\title{
Real-time traffic congestion detection based on frame difference function and virtual loop
}

\author{
Fei Liu', a ${ }^{*}$, Zhiyuan Zeng ${ }^{1, b}$ and Rong Jiang ${ }^{2, c}$ \\ ${ }^{1}$ Hubei Key Laboratory of Digital Valley Science and Technology, Huazhong University of Science \\ and Technology, Wuhan, Hubei Province, China \\ ${ }^{2}$ Huawei Corporation, China \\ acchengru2003@hust.edu.cn, bzengzy@hust.edu.cn, ‘649888951@qq.com
}

Keywords: traffic congestion detection; image processing; intelligent transport system

\begin{abstract}
Traffic congestion has become one of the major concerns of policy-makers in modern metropolises. Accurate real-time traffic congestion alert is of great importance for alleviating congestion. In this paper, we propose a fast, unsupervised, video-based approach using average frame difference function (AFDF) and virtual loop to identify real-time traffic congestion. This novel method calculates the frame differences of specified order in a loop area to determine the speed of the vehicles. The proposed method has been integrated in the intelligent transport system (ITS) of Wuhan City, China for testing, and results show that the method is efficient and robust for real-time traffic congestion detection..
\end{abstract}

\section{Introduction}

As the biggest city in Central China with more than 1.7 million vehicles registered, Wuhan is suffering from severe traffic congestion problems like many of the other big cities of the developing nation. The demands for traffic congestion alert is urgent for traffic management. Besides, accurate and real-time traffic information including the traffic congestion status is one of the fundamental parts of ITS. In this paper, we focus only on the status of traffic congestion, which means that the output of the proposed method is whether the traffic is congested or not in a certain lane section. The algorithm can be easily applied to multi-lanes applications.

Many researchers have dedicated their effort to construct the congestion detection algorithm in recent years. Vehicle-to-infrastructure (V2I) methods such as [1] and vehicle-to-vehicle (V2V) methods such as [2] both require the on board units or sensors which make it less feasible and efficient when huge numbers of vehicles are taken into account. Compared with V2I or V2V, video-based approaches are less expensive and is easy to maintain. Most of the existing video-based approaches [3-6] have not kept a good balance between accuracy and real-time performance. In this paper, we combine the concept of virtual loop which is inspired by magnetic loop sensor [7] and a modified frame difference method to detect traffic congestion. This method only requires a day-night infrared camera that is able to work 24 hours a day and a computer that has the access to the camera. It is rather cheap and is easy to maintain. And it only engages small amount of pixel-wise calculation which ensures the system's ability to run with a great speed. Tests show that the method responses quickly and the accuracy of congestion detection is satisfactorily high.

\section{Virtual loop and average frame difference function}

The average frame difference function (AFDF). It stands to reason that traffic congestion can be interpreted as a state that little difference exists between adjacent frames, which makes frame difference a potential criteria for traffic congestion identification. Instead of calculating the whole frame, a user-defined virtual loop is used for calculation, which is shown in Fig. 1.

The camera is mounted up above the one-way road, and at the bottom of each lane a virtual loop is defined. The red rectangles are virtual loops, and yellow envelops are the corresponding region of interest (ROIs) of three different lanes. When the traffic status of each of the lanes is known, then the 
traffic status of the whole road can be determined. Thus the point of detecting congestion is to determine the state of each single lane which can be inferred from the changes of AFDF. The $n$th order AFDF is defined as follows:

$$
f(i)=\operatorname{mean}\left\{[I(i)-I(i-n)]_{l o o p}\right\}
$$

$I(i)$ is the $i$ th frame of the video. The subscript loop means the ROI regions of the calculation. The region out of the loop will not be considered. mean is the average operator. We calculate the $f(i)$ for all the frames behind the nth frame.

Fig. 2 is obtained by calculating the AFDF in the region of the middle loop after every 2, 3, 4, 5, 6 frames with respect to frame time. The source frames are converted to gray images for calculation and essential preprocessing actions such as image smoothing and image de-nosing have been applied to the frames. The source video is about 50s long. The video starts with a normal traffic state and then falls into a traffic jam till the end. As the video shows, three vehicles intrude the area of the virtual loop and the former two leave immediately except that the speed of the first vehicle is much bigger than the second one. The third vehicle leaves at about 25 seconds later and another one drives in in one or two seconds. Then almost no vehicles move.

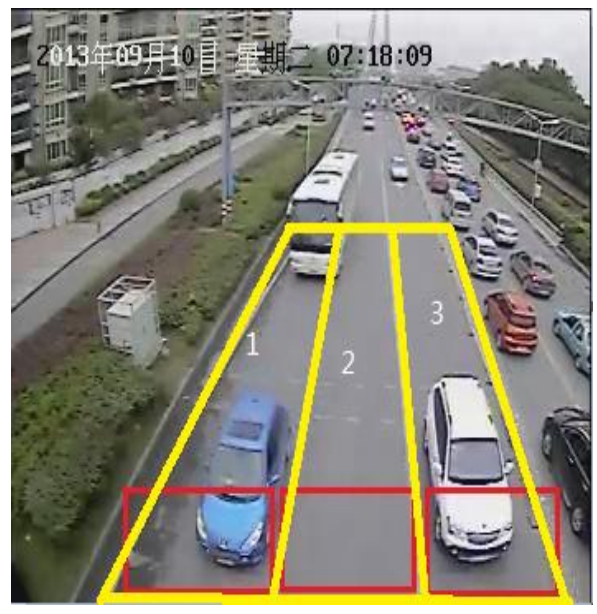

Figure 1. Virtual loops and ROIs
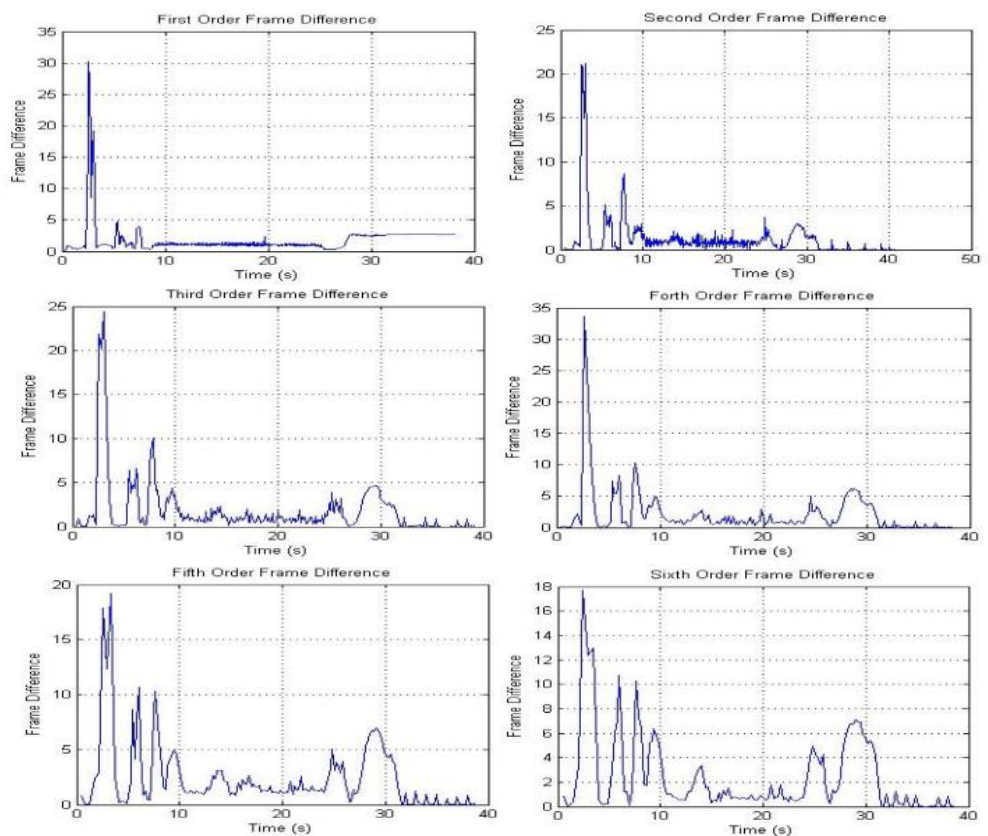

Figure 2. AFDF of different orders

As Fig. 2 shows, the whole actual process has a strong connection with the AFDF especially the 3rd and the higher order AFDFs. The basic point is, higher values of AFDF indicate good traffic conditions and the values below a certain level correspond to congestion or free road. There is no denying the AFDF is a good indicator of whether the vehicles are floating. And after a thoroughgoing study of the results shown in Fig 2, there are some basic verdicts about the AFDF that can be concluded:

- The move of vehicles produces an impulse-like change in the AFDF in all orders while the period of traffic jam corresponds to the flat area of the function.

- First order AFDF is sensitive to fast moving vehicles but is very dull to slow ones. The boundary between congestion and free traffic is sharply clear. But mistakes exist when vehicles move a very small distance in congested traffic.

- The response of higher order AFDF to slow changes in the video is stronger than that of the first orders'. Higher order brings higher detail resolution. We can even find the exact time that a vehicle drives into or out of the virtual loop from the sixth order AFDF.

- The fourth order AFDF is so different from the other orders'. It has strong response to fast moving vehicles than the lower-orders' and preserves a relative high detail resolution. 
- The sixth order AFDF and the fifth order AFDF are very similar to each other. The fifth order AFDF has a lot disturbances inside the impulse.

As we can see, for the tested video the fourth order AFDF and the sixth order AFDF are better choices for detecting congestion. They have relatively strong responses to fast moving vehicles and have little disturbances. Both of them have excellent performances when applied in congestion detection. Actually we can combine the AFDF of different orders' in the calculation to make the result more reliable. In this paper, we use a single sixth order AFDF for simplicity.

\section{Free road detection.}

It is noteworthy that when the road is free of vehicles, the AFDF will keep at a level near zero which will be recognized as congestion. So before applying AFDF for detecting congestion, we have to determine whether there are vehicles in the loops. The difference between an empty road and a road be filled with congested vehicles lies in their pixel distributions. The pixel distribution of a free road region is highly uniform while the one of an occupied road differs much. Variance is one of the efficient indicators to specify the difference of the two distributions. Table 1 is obtained by calculating the variances of the ROI regions of a single lane for occasions with free road and occupied road.

Table1 Variance of ROI of different occasions

\begin{tabular}{|c|c|c|c|c|c|}
\hline \multirow{2}{*}{ Day/Night } & \multirow{2}{*}{$\begin{array}{l}\text { Rainy/Not } \\
\text { rainy }\end{array}$} & \multicolumn{2}{|c|}{ Variance of ROI (V) } & \multicolumn{2}{|c|}{$I(V)=10^{\mathrm{V} / 100}$} \\
\hline & & Occupied & Free & Occupied & Free \\
\hline \multirow{2}{*}{ Day } & Rainy & 2482.18 & 749.98 & 303.51 & 5.62 \\
\hline & Not Rainy & 1339.61 & 167.21 & 25.86 & 1.49 \\
\hline \multirow{2}{*}{ Night } & Rainy & 2261.43 & 985.9 & 182.57 & 6.68 \\
\hline & Not Rainy & 1619.86 & 534.57 & 41.67 & 3.47 \\
\hline
\end{tabular}

Notice: The videos of the night in this paper are taken with efficient auxiliary lighting system.

As TABLE I shows, the image variance of the free road is much smaller than the occupied road. Based on this point, we can easily distinguish the false congestion from the real one. To reinforce the variance difference between the two occasions, we define a function of real variance $\mathrm{V}$ as follows:

$$
I(V)=10^{V / 100}
$$

$\mathrm{I}(\mathrm{V})$ is also calculated in Table 1 . It can be inferred from the table that $\mathrm{I}(\mathrm{V})$ is an efficient indicator of whether the ROI is occupied by vehicles or is free. The threshold value of $\mathrm{I}(\mathrm{V})$ can be chosen from range $[7,20]$ in this case. An $\mathrm{I}(\mathrm{V})$ value below the threshold specifies a free lane. Besides, as we can see from Table 1, I(V) is strongly connected with weather and lighting conditions. Thus we can apply different threshold values in different occasions. For example, the threshold must be set a bit higher in nighttime than in the daytime. A rainy occasion needs a higher threshold value too.

\section{Calculation Procedure}

As described above, the basic way of detecting congestion is to check the average value of AFDF in a certain period. The period should encompass at least the width of an impulse, which is about 3 seconds or 8 frames long as shown in Fig. 3. 

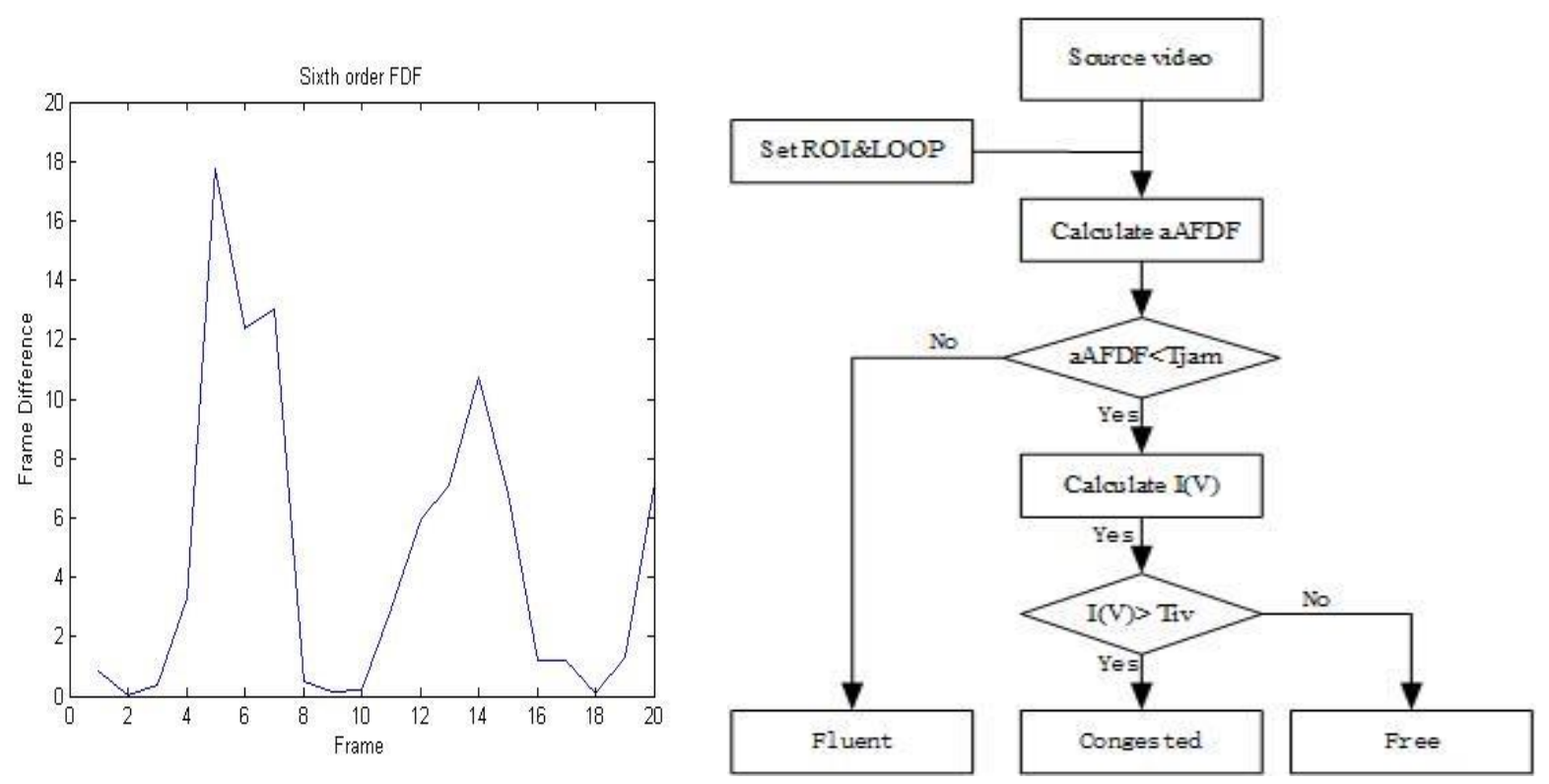

Figure 3. Sixth order AFDF with respect to frame sequence

Fig. 4 Calculation process

Fig. 3 is obtained by calculating the AFDF with respect to frame sequences instead of time. It can be inferred from Fig. 3 that 8 is a potential unit for calculation, which involves a range of 48 frames. In this paper, we adopt 10 as a calculation unit. In practical applications using more units such as 2 units which involve a range of 120 frames will produce more precise result of traffic state prediction. We have to be aware of that the calculation unit is not the same for different occasions. The speed limit specified by the road management bureau determine that value.

Suppose $a A F D F$ is the average value of the AFDF in a certain range. Tjam is the determination threshold. Let $I(V)$ be the variance function and $T i v$ be the variance threshold. The whole procedure of real-time traffic detection can be summarized as Fig. 4. The threshold value Tjam can be obtained by calculating the average AFDF of a longer period of congestion and be updated when a new jam happens. We adopt the value of 2 for testing. What is worth noticing is that the threshold values of both $a A F D F$ and $I(v)$ should be determined based on experimental results in different road and lighting conditions. The best way is to set certain update rules for the thresholds.

\section{Results}

We randomly picked 2 sample cases from candidate videos for testing. Both videos are 45 seconds long and have the processes of congested-to-uncongested occasion or (and) the uncongested-tocongested occasion. And we deal with only one lane in this experiment as the single lane cases can be easily extended to multi-lane cases. We recorded the time of every event from observation for reference.

Table 2 Traffic congestion detection

\begin{tabular}{|l|l|l|l|}
\hline \multicolumn{2}{|l|}{ Events/Time } & Observed(s) & Estamated(s) \\
\hline \multirow{4}{*}{ Case 1 } & Free & Begining \\
\cline { 2 - 4 } & Into congestion & 11 & 11.56 \\
\cline { 2 - 4 } & Off congestion & 20 & 19.40 \\
\cline { 2 - 4 } & Into congestion & 37 & 36.92 \\
\hline \multirow{4}{*}{ Case 2 } & Congestion & Begining \\
\cline { 2 - 4 } & Off congestion & 13 & 12.6 \\
\cline { 2 - 4 } & Into congestion & 27 & 27.4 \\
\cline { 2 - 4 } & Off congestion & 34 & 34.0 \\
\hline
\end{tabular}

Table 3 Result of free road detection

\begin{tabular}{|l|l|l|l|l|}
\hline Case & Item & Frames & Recognized & Error \\
\hline \multirow{2}{*}{$\begin{array}{l}\text { Case } \\
\text { 1:Day }\end{array}$} & Occupied & 22 & 21 & 1 \\
\cline { 2 - 5 } & Free & 12 & 12 & 0 \\
\hline \multirow{2}{*}{$\begin{array}{l}\text { Case } \\
\text { 2:Night }\end{array}$} & Occupied & 9 & 9 & 0 \\
\cline { 2 - 5 } & Free & 13 & 13 & 0 \\
\hline \multirow{2}{*}{$\begin{array}{l}\text { Case } \\
\text { 3:Rainy }\end{array}$} & Occupied & 6 & 6 & 0 \\
\cline { 2 - 5 } & Free & 11 & 11 & 0 \\
\hline
\end{tabular}


Based on the thoughts described above, we have written a testing program in MATLAB language. We run the program for both chosen videos and saved the results into files. The threshold value is set to be 50. Table 2 shows the results collected from both observation and the program

As we can see from Table 2, the program gives reliable and precise results. Besides detecting the congested traffic, the program produces the exact time of the congestion events.

To verify the free road detection method, we picked 3 videos of typical occasions and randomly choose a certain number of frames to do the variance calculation. The threshold value is set to 7 for all cases. The results are shown in Table 3.

As Table 3 shows, our method can recognize almost all occasions correctly. In case 1, the false recognition reasoned from a vehicle whose color is very similar to the road surface which is very rare in actual application. Threshold can be set to lower values such as 4 or 5, but a value as big as possible can make sure that the bicycles or motorcycles be excluded from further calculation.

As shown above, the method proposed can detect congestion and the time it happens and disappears efficiently and robustly. The method is able to record 24-hours-a-day the real-time traffic status, which in this paper is categorized into free, fluent, congested. Given the output of AFDF data, we can also determine the overall speed of the traffic.

\section{Conclusions}

This paper proposed a lightweight real-time traffic surveillance system based on video image processing. Our system, which is able to detect traffic congestion with millisecond-level precision, is very helpful for the traffic management department to work effectively. And the system requires just a day-night camera and a host computer which makes its installation and maintenance simply and conveniently. As the experiment shows, the system is also robust and efficient. It can work at rainy weather condition and at night. The further work including searching for a better mechanism to determine the most appropriate threshold values for both AFDF and free road detection. Dynamic threshold values can increase the accuracy rate of the detection. Besides, more types of frame difference function could be tested to find a better indicator for congestion detection.

\section{References}

[1] Wang C, Tsai H M. Detecting urban traffic congestion with single vehicle[C], Connected Vehicles and Expo (ICCVE), 2013 International Conference on. IEEE, 2013: 233-240.

[2] Milojevic M, Rakocevic V. Distributed Vehicular Traffic Congestion Detection Algorithm for Urban Environments[J]. 2013

[3] L. Li, C. Long, H. Xiaofei, and H. Jian, "A Traffic Congestion Estimation Approach from Video Using Time-Spatial Imagery", Intelligent Networks and Intelligent Systems, 2008. ICINIS '08. First International Conference on, 2008, pp. 465-469.

[4] H. Glasl, D. Schreiber, N. Viertl, S. Veigl, and G. Fernandez, "Video based Traffic Congestion Prediction on an Embedded System", Intelligent Transportation Systems, 2008. ITSC 2008. $11^{\text {th }}$ International IEEE Conference, 2008, pp. 950-955.

[5] Porikli F, Li X. Traffic congestion estimation using HMM models without vehicle tracking[C], Intelligent Vehicles Symposium, IEEE, 2004: 188-193.

[6] Nidhal A, Ngah U K, Ismail W. Real time traffic congestion detection system, 2014 5th International Conference on Intelligent and Advanced Systems (ICIAS)

[7] J Gajda, R Sroka, M Stencel, A Wajda. A vehicle classification based on inductive loop detectors [M]. Instrumentation and Measurement Technology Conference, Volume 1(2001) 460-64. 